\title{
Article \\ The Role of the Western Diet and Oral Microbiota in Parkinson's Disease
}

\author{
Barbara Zapała ${ }^{1, *}$, Tomasz Stefura ${ }^{2}$ (D) Tomasz Milewicz ${ }^{3}$, Julia Wątor ${ }^{4}$, Monika Piwowar ${ }^{5}$, \\ Magdalena Wójcik-Pędziwiatr ${ }^{6}$, Magdalena Doręgowska ${ }^{6}$, Alicja Dudek ${ }^{2}$, Zuzanna Jania ${ }^{1}$ \\ and Monika Rudzińska-Bar ${ }^{6}$ (D)
}

1 Department of Clinical Biochemistry, Jagiellonian University Medical College, 31-066 Krakow, Poland; Zuzannajania@gmail.com

2 2nd Department of General Surgery, Jagiellonian University Medical College, 31-008 Krakow, Poland; tomasz.stefura@gmail.com (T.S.); ala.ddudek@gmail.com (A.D.)

3 Department of Gynaecological Endocrinology and Gynaecology, Jagiellonian University Medical College, 31-501 Krakow, Poland; tomasz.milewicz@uj.edu.pl

4 Faculty of Medical Sciences in Zabrze, Medical University of Silesia, 41-800 Katowice, Poland; julia.katarzyna.wator@gmail.com

5 Department of Bioinformatics and Telemedicine, Faculty of Medicine, Jagiellonian University Medical College, 31-034 Krakow, Poland; monika.piwowar@uj.edu.pl

6 Department of Neurology, Andrzej Frycz Modrzewski Krakow University, 30-705 Krakow, Poland; m.pedziwiatr@szpitaljp2.krakow.pl (M.W.-P.); mdoregowska@afm.edu.pl (M.D.); mrudzinska@afm.edu.pl (M.R.-B.)

* Correspondence: barbara.zapala@uj.edu.pl

check for updates

Citation: Zapała, B.; Stefura, T.; Milewicz, T.; Wątor, J.; Piwowar, M.; Wójcik-Pędziwiatr, M.; Doręgowska, M.; Dudek, A.; Jania, Z.;

Rudzińska-Bar, M. The Role of the Western Diet and Oral Microbiota in Parkinson's Disease. Nutrients 2022, 14, 355. https://doi.org/10.3390/ nu14020355

Academic Editor: Luigi Barrea

Received: 2 November 2021

Accepted: 12 January 2022

Published: 14 January 2022

Publisher's Note: MDPI stays neutral with regard to jurisdictional claims in published maps and institutional affiliations.

Copyright: (C) 2022 by the authors. Licensee MDPI, Basel, Switzerland. This article is an open access article distributed under the terms and conditions of the Creative Commons Attribution (CC BY) license (https:// creativecommons.org/licenses/by/ $4.0 /)$.

\begin{abstract}
The type of diet not only affects the composition of the oral microflora but is also one of the more critical factors associated with an increased risk of Parkinson's disease, PD. This study compared diet preferences and oral microbiota profiles in patients with PD vs. healthy controls. This study compared the oral microbiota composition of 59 patients with PD and 108 healthy controls (without neurodegeneration) using $16 \mathrm{~S}$ rRNA gene amplicon sequencing. According to results, oral microbiota in patients with PD is different compared from healthy controls. In particular, decreased abundance of Proteobacteria, Pastescibacteria, and Tenercutes was observed. The oral cavity of patients with PD was characterized by the high relative abundance of bacteria from the genera Prevotella, Streptococcus, and Lactobaccillus. There were also differences in food preferences between patients with PD and healthy controls, which revealed significantly higher intake of margarine, fish, red meat, cereals products, avocado, and olives in the patients with PD relative to healthy controls. Strong positive and negative correlations between specific food products and microbial taxa were identified.
\end{abstract}

Keywords: Parkinson's disease; the Western diet; oral microbiota

\section{Introduction}

PD is the second most common neurodegenerative disorder, affecting up to $1 \%$ of the population above 60 and $4-5 \%$ of those above 80 [1,2]. Men are about 1.5 times more likely than women to develop PD among the patients. Due to the acceleration of the aging of Western populations, incidence above the current 1 -in-800 is predicted [3]. When considering that less than $10 \%$ of PD is associated with specific genetic changes, researchers are still looking for environmental risk factors causing the disease. Indeed, findings regarding the role of dietary factors in PD are conflicting. There is some evidence for a protective role of caffeine and a deleterious role of dairy products. Recently, the MIND diet, which is based on the Mediterranean and DASH diets, has also been associated with a delayed onset of PD [4-6]. The most popular in Europe, the Western diet, is characterized by high caloric intake of energy-dense foods, high in saturated and omega-6 (w6) fatty acids, refined sugars, excessive salt intake, and low consumption of omega-3 (w3) fatty 
acids and fiber. This diet, mainly enriched in high quantities of animal saturated fats, canned fruits and vegetables, soda, fried foods, beef, ice cream, and cheese, has been widely associated with an increased risk of developing PD [7-10]. On the other side, the Mediterranean diet correlates with decreased risk of developing PD and its components, such as fresh vegetables and fruit, nuts, seeds, fish, olive oil, wine, fresh herbs, and spices. Furthermore, consumption of flavonoid-rich foods (e.g., tea, berry fruits, apples, red wine, and orange juice) seem to be associated with a lower risk of developing PD.

Polyunsaturated fatty acids (PUFA) were inversely correlated with PD development [11-14]. Additionally, several reports have even shown that higher consumption of $\omega 3$ fatty acids is associated with a lower risk of PD development, and this has provoked suspicion that $\omega 3$ fatty acids influence brain function [11-14]. There are multiple mechanisms by which a diet can impact the body's homeostasis, for example, by the direct influence of dietary components as vitamins or fats. However, the most intriguing point now is the indirect influence of diet components on the human microbiome [13].

The intestinal microbiota was significantly different in patients with PD compared to healthy controls [15-17]. However, it is worth emphasizing that gut microbiota is disrupted among patients diagnosed with PD, and disruptions in oral and even nasal microbiota profiles may influence the development and progression of the disease. Throughout life, many factors influence oral microbiota, including environmental factors, dietary habits, host genetics, hygiene practice, and medications. Although oral microbial communities are highly variable, prior evidence has demonstrated that microbiome perturbations in the oral cavity can influence health and provoke disease [15-17]. For example, it has been reported that opportunistic pathogens can provoke diseases such as pneumonia, and such pathogens were observed to be more abundant in the mouths of people with PD than in healthy individuals. Thus, changes in oral cavity bacteria in patients with PD could influence disease symptoms affecting the mouth, particularly drooling and difficulty swallowing, which are well-known symptoms of PD [15-17]. The opportunistic pathogens may be hazardous under certain circumstances. For example, in PD, oral health is poorer than in healthy individuals [18]. PD patients have difficulties flossing or brushing the teeth, which, when combined with increased drooling and difficulty swallowing, provoke plaque building in the mouth and cause the formation of a fertile breeding ground for bacteria [18]. It was demonstrated that Streptococcus pneumoniae was an opportunistic pathogen responsible for provoking pneumonia. In PD patients, symptoms such as aspiration pneumonia, caused by inhaling food, drinks, vomits, or saliva into the lungs, are a very common cause of death [18]. Moreover, it has been observed that PD patients with trouble swallowing and excess drooling were characterized by a significantly higher abundance of $S$. pneumoniae in the oral cavity. What was very important in the study reported by Rozas et al. was that there were no statistically significant differences observed in oral hygiene habits between the patients with PD and healthy controls [18]. These results strongly suggested that poor oral health observed in PD patients is not caused by the reduced frequency or efficiency of oral hygiene but rather is linked to disease-specific factors resulting in increased abundance of certain bacteria, which can initiate an oral disease [18]. There are much fewer reports describing oral microbiota concerning PD. However, it has been shown that there are differences in oral microbiome profiles in patients with PD compared to healthy controls. Previous studies report that oral microbiota in patients with PD is much more enriched in opportunistic pathogens when compared to healthy controls [15-17].

This study compared diet preferences and oral microbiota profiles in patients with PD vs. healthy controls.

\section{Materials and Methods}

We performed a cross-sectional, descriptive, and comparative study including 59 patients with PD and 108 healthy controls (without neurodegeneration), ranging from 51 to 82 years old. Patients with PD were recruited from one of the hospital centers in Krakow, Poland, and healthy controls were volunteers. In the group of patients with PD, the stage 
of disease progression was assessed using Hoehn and Yahr staging ( $\mathrm{H} \& \mathrm{Y}$ ) and Movement Disorder Society Unified Parkinson's Disease Rating Scale part III (MDS-UPDRS Part III) score during ON time. The patients with PD were excluded from this study when diagnosed with other neurological diseases such as systemic or neurologic infections, inflammatory or autoimmune diseases, atypical parkinsonism syndrome, and vascular parkinsonism. The exclusion criterium in that group also was brain surgery, concomitant psychiatric diseases such as schizophrenia or bipolar disorder, preliminary baseline evaluations. Patients with confirmed or suspected gastrointestinal malignant tumors or other gastrointestinal diseases were excluded. Patients with PD with a medication history of using antibiotics or probiotics over the last three months or therapy based upon steroids, non-steroidal anti-inflammatory drugs, or history of gastrointestinal surgery (e.g., gastro-resection or major intestinal surgery) did not participate in this study. Smokers were also excluded from the study. Participants with complete dentures or removable dental prostheses, or the presence of fewer than 16 natural teeth, were excluded from the study. The cognitive and functional status were scored using the Mini-Mental State Examination (MMSE) and the Montreal Cognitive Assessment (MoCA).

Additionally, computed tomography scanning or magnetic resonance imaging was performed in PD patients to exclude vascular parkinsonism. The detailed demographic data and medical history were collected using a set of questionnaires. The healthy volunteers were matched for age and were enrolled in the study based on exclusion criteria: any medical history for neurological, immunological, gastrointestinal diseases, and smoking.

All participants received a questionnaire to analyze their food preferences. The questionnaire consisted of two parts and was performed before sampling. The first part was used to determine anthropometric parameters such as body weight, height, age, gender, socioeconomic, educational status, and medical history. The second section included 33 foods, including ingredients typical of the Western and Mediterranean diets. The second part also included questions about preparing food such as boiling, steaming, baking, grilling, and frying. Food items were listed under significant food groups such as vegetables, fruit, cereals, legumes, milk, dairy products, meat and fish, eggs, oils, fats, drinks (including alcohol drinks, coffee, and tea), and snacks or fast food. Respondents reported the frequency of consumption of each food product as always, above three times per day, twice a day, once a day, 4-6 times per week, 2-3 a week, once a week, monthly, or never.

Oral samples were collected with BactiSwab ${ }^{\mathrm{TM}}$ NPG Collection and Transport System (ThermoFisher Scientific, Waltham, MA, USA), which has a unique cap design to reduce contamination during sample collection and during the DNA isolation stage, which was critical for this microbiome studies. The inner surfaces of the buccal mucosa, tongue, and hard palate were rubbed with the swab several times for at least $10 \mathrm{~s}$, then the swab samples were transferred to the tubes and immediately stored at $-80^{\circ} \mathrm{C}$. Before oral sampling, all participants were well informed about refraining from eating or drinking for at least $30 \mathrm{~min}$ before the sample collection. The commercially available QIAamp BiOstic Bacteremia DNA Kit (QIAGEN, Hilden, Germany) extracted bacterial genomic DNA. The bacterial genomic DNA was extracted from buccal swabs. During the extraction, all steps followed the manufacturer's protocol. The extracted DNA was estimated quantitatively and qualitatively using spectrophotometer NanoDrop ND-1000 (Thermo Electron Corporation, West Palm Beach, FL, USA) and fluorometer Qubit 4 (Invitrogen, Waltham, MA, USA). Then, all isolates were stored at $-20{ }^{\circ} \mathrm{C}$ until further analysis. Blood samples were collected from all study participants to measure biochemical parameters such as triglyceride, total cholesterol, high-density lipoprotein (HDL), low-density lipoprotein (LDL), alanine aminotransferase (ALT), aspartate aminotransferase (AST), white blood cells (WBCs), and red blood cells (RBCs) platelets (PLT).

A sequencing library of the $16 \mathrm{~S}$ rRNA gene V3 and V4 regions was constructed using gene-specific primers adapted from the Klindworth et al. publication [19]. The libraries were prepared under the protocol for Preparing 16S Ribosomal RNA Gene Amplicons for the Illumina MiSeq System. The PCR-based amplification was performed following 
the protocol of the manufacturers of the KAPA HiFi HotStart ReadyMix (ROCHE, Basel, Switzerland). Amplification was performed under the following thermocycling: $95{ }^{\circ} \mathrm{C}$ for $1 \mathrm{~min}, 55^{\circ} \mathrm{C}$ for $1 \mathrm{~min}$, then $72{ }^{\circ} \mathrm{C}$ for $1 \mathrm{~min}$ for 30 cycles, and a final extension step at $72{ }^{\circ} \mathrm{C}$ for $5 \mathrm{~min}$. The PCR products were then indexed with specific sequencing adapters using Nextera XT Index Kit v2 from Illumina. The indexing step was performed in a thermocycler using the following steps: $95^{\circ} \mathrm{C}$ for $3 \mathrm{~min}$, eight cycles of $95^{\circ} \mathrm{C}$ for $30 \mathrm{~s}, 55^{\circ} \mathrm{C}$ for $30 \mathrm{~s}$, $72{ }^{\circ} \mathrm{C}$ for $30 \mathrm{~s}$, and a final extension at $72{ }^{\circ} \mathrm{C}$ for $5 \mathrm{~min}$ and hold at $4{ }^{\circ} \mathrm{C}$. The sequencing was performed on the MiSeq instrument (Illumina, San Diego, CA, USA) using a $300 \times 2$ V3 Kit and PhiX Control V3 from Illumina. The Qubit 4.0 Fluorometer (Invitrogen, Waltham, MA, USA) and Bioanalyzer (Agilent, Santa Clara, CA, USA) were used to assess the integrity and size ( $\sim 630 \mathrm{bp})$ of amplicons. Prior to sequencing, the amplicons were pooled in equimolar concentrations. The raw reads of $16 \mathrm{~S}$ rRNA gene sequences generated as FASTQ formats were filtered using the Illumina16S Metagenomics workflow to obtain high-quality reads. Then, the high-quality sequences were clustered into operational taxonomic units (OTUs) at $99.9 \%$ identity based on the Greengenes Database and the algorithm with the highperformance implementation of the Ribosomal Database Project (RDP) classifier, which was described by Wang Q. et al. in 2007 [20]. QIIME 2.0 software with Python scripts was applied to calculate alpha- and beta-diversity [21]. Alpha-diversity was measured based on the sequence similarity at $97 \%$ level. The number of unique OTUs found in each sample was assessed to present the richness. The results were presented as ACE and Chao1 indices. The Shannon, Simpson, and Fisher estimators measured both the richness and evenness within individual samples and both experimental groups [21,22]. Beta-diversity, calculated as the distance and dissimilarities between microbial communities, was determined using Jaccard, Bray-Curtis, and Jensen-Shannon Divergence indices. These results were visualized by principal coordinate analysis (PCoA) [23]. The statistically significant differences based on beta-diversity of the whole microbiome structure between patients with PD and healthy controls were calculated using a per-mutational multivariate analysis of variance (PERMANOVA). LEfSe and MicrobiomeAnalyst were then applied to perform the clustering and statistical analysis [24,25]. The linear discriminant analysis (LDA) effect size from LEfSe was adapted to indicate the most statistically significant features of oral microbiota. The discovered microbial biomarkers with statistical significance and biological relevance were de-scribed based on the normalized relative abundance matrix, the Kruskal-Wallis rank-sum test, the significant alpha at 0.05, and the effect size threshold of 2. The median abundance and the non-parametric Wilcoxon Rank Sum test were used to discover statistically significant taxonomic differences between microbial communities and abundance profiles of two experimental groups [26]. Raw data of the food preferences from questionnaires were statistically analyzed with the statistical environment $\mathrm{R}$ version 3.6.0, PSPP software, and MS Office 2019. The descriptive statistics were prepared, and frequency distribution was calculated and presented as means and standard deviations (SD). Adjustments were applied for age, sex, BMI, and kilocalories. Multivariate analysis was performed to determine the statistically significant differences of the means when comparing both groups' results. The Chi-square and Fisher's tests were used to analyze the variables on the nominal scale. The Student $t$-test $t$, the Mann-Whitney $U$, and the Kruskal-Wallis test were adapted for the quantitative variables. A $p$-value of $<0.05$ was considered significant.

The study was performed following the highest ethical standards of the hospital (in which the patients were recruited), national guidelines, and the Helsinki Declaration. All protocols for the study were approved by the Ethics Committee of Jagiellonian University Medical College (approvement number 1072.6120.267.2019) and written informed consent was obtained from each subject before enrollment. 


\section{Results}

\subsection{Participants}

Patients with PD ranged from 55 to 82 years, whereas the healthy controls ranged from 51 to 82 years. The average age of patients with PD was $69 \pm 7$ years, and $24(41 \%)$ were female. The healthy controls' average age was $64 \pm 7$ years, and 63 (58\%) were female. In the group of patients with PD, the mean BMI was $26.3 \pm 3 \mathrm{~kg} / \mathrm{m}^{2}$, and in the control group, it was $25.8 \pm 4 \mathrm{~kg} / \mathrm{m}^{2}$, which fall to within the overweight range but not obese. The average duration of the disease totaled 7.32 years (standard deviation 6.67). In the analysis, patients with PD included in the research group showed a grade of 2.04 on the five-point Hoehn and Yahr scale (standard deviation 0.71). The Mini-Mental State Examination scored 26.33 (standard deviation 1.51), and the Montreal Cognitive Assessment scale scored 21.8 (5.15). Both indicators denoted mild cognitive impairment among patients with PD. No significant differences in the analyzed clinical parameters and anthropometric characteristics were observed between patients with PD and healthy controls (Tables 1 and 2).

Table 1. Characteristics of patients with PD and age-matched healthy controls.

\begin{tabular}{|c|c|c|c|c|c|c|c|c|c|}
\hline & Group & $\mathbf{N}$ & $\mathbf{M}$ & SD & Min & Max & Q25 & Me & Q75 \\
\hline \multirow{2}{*}{ Age (years) } & PD & 59 & 69.34 & 7.07 & 55.00 & 82.00 & 65.00 & 68.00 & 75.00 \\
\hline & Controls & 108 & 64.21 & 10.23 & 51.00 & 82.00 & 54.50 & 62.00 & 71.50 \\
\hline \multirow{2}{*}{ Weight (kg) } & PD & 59 & 76.91 & 14.52 & 48.00 & 103.00 & 67.25 & 79.00 & 88.25 \\
\hline & Controls & 108 & 72.95 & 13.38 & 47.00 & 118.00 & 65.00 & 70.00 & 80.00 \\
\hline \multirow{2}{*}{ Height (m) } & PD & 59 & 1.70 & 0.11 & 1.50 & 1.86 & 1.62 & 1.70 & 1.78 \\
\hline & Controls & 108 & 1.68 & 0.08 & 1.50 & 1.90 & 1.64 & 1.66 & 1.75 \\
\hline \multirow{2}{*}{ BMI } & PD & 59 & 26.33 & 2.92 & 19.23 & 32.30 & 25.14 & 26.53 & 28.16 \\
\hline & Controls & 108 & 25.81 & 3.75 & 18.36 & 33.22 & 22.92 & 25.56 & 28.17 \\
\hline
\end{tabular}

$\mathrm{N}$-numbers; M-mean; SD—standard deviation; Min-minimum; Max-maximum; Q—quartile; Me-median.

Table 2. The results of urine analysis in patients with PD.

\begin{tabular}{ccc}
\hline Analysis & Result & Reference Values \\
\hline Urine color & yellow / dark amber $(24 / 3)$ & yellow \\
Clarity & clear/clour $(22 / 5)$ & normal \\
Acidity & normal/acidic $(17 / 10)$ & $1020 \pm 0.02$ \\
Specific gravity & $1020 \pm 0.02$ & negative \\
Glucose & negative & negative \\
Ketones & negative & negative \\
Nitrates & negative & negative \\
Bilirubin & negative & negative \\
Urobilinogen & negative & $\leq 3$ \\
Blood & $\leq 3$ red blood cells & $\leq 2$ \\
Red blood cells & $\leq 2$ RBCs $/$ hpf & $\leq 2-5$ \\
White blood cells & $\leq 2-5$ WBCs $/ \mathrm{hpf}$ & $\leq 150$ mg/day \\
Protein & $\leq 150$ mg/day & negative \\
Squamous epithelial cells & negative & negative \\
Casts & negative & negative \\
Crystals & negative & none \\
Bacteria & none/present $(22 / 5)$ & none \\
Yeast & none &
\end{tabular}

\subsection{Differences in Diet Preferences between Patients with PD and Healthy Controls}

Patients with PD consumed more margarine $(p<0.007)$, breakfast cereal products $(p<0.030)$, and avocado and olives $(p<0.001)$. In patients with PD, the fish intake was greater than in the controls $(p<0.001)$. However, the patients with PD more frequently 
consumed red meat than did the healthy controls $(p<0.015)$ (Figure 1 and Table 3$)$. Lower consumption of thick groats $(p<0.001)$, dark bread $(p<0.001)$, peanuts $(p<0.001)$, juices $(p<0.004)$, soy $(p<0.028)$, fresh fruits $(p<0.008)$, and cross vegetables $(p<0.001)$ was observed in patients with PD when compared to the healthy controls (Figure 1 and Table 4 ). Sweetened drinks intake was lower in patients with PD $(p<0.012)$ compared with the healthy controls.

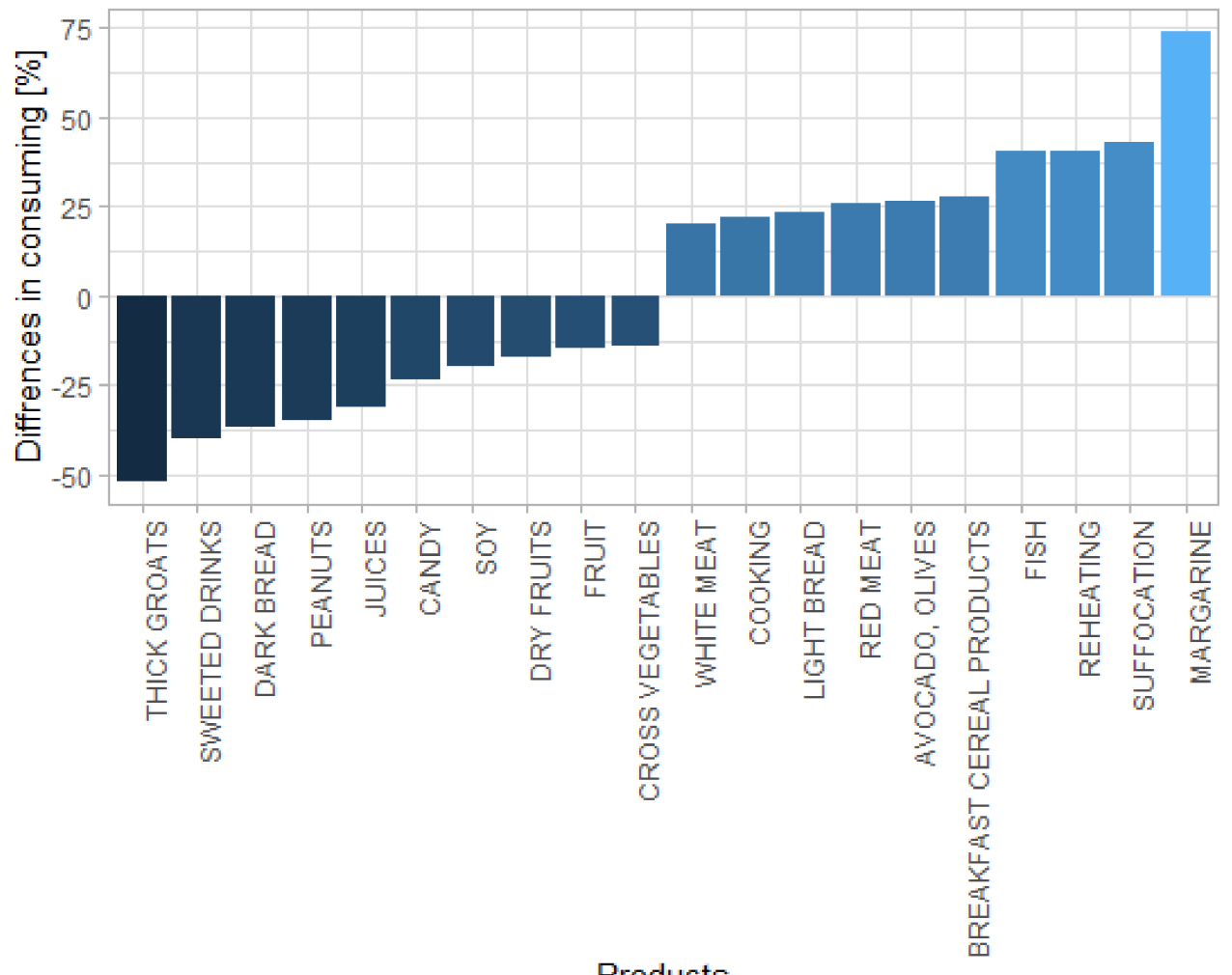

Products

Figure 1. Differences in food preferences between patients with PD and the healthy controls.

Table 3. The frequency of products consumed by patients with PD compared to the healthy controls. Table 3 shows the products with higher intake in patients with PD compared to healthy controls.

\begin{tabular}{ccc}
\hline Product & Differences (\%) & $p$-Value \\
\hline Margarine & 74.07 & 0.007 \\
\hline Fish & 40.24 & 0.001 \\
\hline Breakfast cereal products & 27.67 & 0.030 \\
\hline Avocado, olives & 26.74 & 0.001 \\
\hline Red meat & 26.04 & 0.015 \\
\hline
\end{tabular}

There were noticed statistical differences only in vitamin $C$ consumption between the patients with PD and the healthy controls. The patients with PD consumed lower doses of vitamin $C$ when compared to the healthy controls $(p<0.01)$. There were no statistical differences between groups concerning vitamin A $(p<0.54)$, vitamin $\mathrm{E},(p<0.67)$, and folic acid $(p<0.14)$. The consumption of high animal fat was higher in patients with PD $(p<0.02)$. There were also statistically significant differences in animal milk intake between the patients with PD and the healthy controls $(p<0.00)$. The patients with PD consumed much fewer carbohydrates than the normal range predicted. The diet of PD patients was also rich in proteins, and they consumed more proteins than was recommended. Moreover, we noticed that the diet of PD patients was a low-fat diet. The average age in the control 
group was $64.21 \pm 10.23$ years; the characteristics of the PD and the healthy controls are shown in Table 1.

Table 4. The frequency of products consumed by patients with PD compared to healthy controls. Significance testing was performed using the R statistical package. Table 4 shows the products with lower intake in the PD group compared to the controls.

\begin{tabular}{ccc}
\hline Product & Differences ${ }^{*} \mathbf{( \% )}$ & $p$-Value \\
\hline Thick groats & -51.96 & 0.001 \\
\hline Sweetened drinks & -39.77 & 0.012 \\
\hline Dark bread & -36.89 & 0.001 \\
\hline Peanuts & -35.13 & 0.001 \\
\hline Juices & -31.14 & 0.004 \\
\hline Canned fruits & -23.31 & 0.055 \\
\hline Soy & -19.94 & 0.028 \\
\hline Dry fruits & -17.28 & 0.285 \\
\hline Fresh fruits & -14.58 & 0.008 \\
\hline Cross vegetables & -14.18 & 0.001 \\
\hline
\end{tabular}

* Minus means deficiency.

\subsection{Microbiota Composition in Patients with PD Group vs. Healthy Controls}

We obtained 3,329,002 reads of the 16S RNA genes. The mean number of reads per sample was 47,557 (367-102,569). The total number of OTU identified was 346.

Alpha-diversity of analyzed samples is presented in Figure 2. Microbiota from the control group revealed significantly higher species richness than the microbiota of patients with PD (Chao1 index $p<0.02$ ACE index $p<0.03$ ). The specific weight of microbiota in the healthy controls is more diverse than in patients with PD.

Analysis of beta-diversity is presented in Figure 2. Microbiota diversity was greater in healthy controls compared to patients with PD (Bray-Curtis F-value: 6.2; R-squared: 0.083558; $p$-value < 0.001; Jensen Shannon Divergence F-value: 10.032; R-squared: 0.12856; $p$-value $<0.001$; Jaccard Index F-value: 4.3828; R-squared: 0.06055; $p$-value $<0.001)$. This is shown in Figure 3.

Bacteroidetes (27\%), Firmicutes (27\%), and Actinobacteria (27\%) constituted the central representation of bacteria in the oral cavity of patients with PD, while Firmicutes (28\%), Proteobacteria (23\%), and Actinobacteria (23\%) occurred mainly in the healthy controls. The oral microbiota composition of the patients with PD and healthy controls are presented in Figure 4.

The analysis of the two most common microbiome clusters (Proteobacteria and Bacteroidetes) showed a much higher number of Proteobacteria in the healthy controls $(p<0.000)$, while Bacteroidetes were significantly more abundant $(p<0.000)$ in the healthy controls. Moreover, a similar number of Proteobacteria were demonstrated in all patients with PD. Figure 5 presents the phylogenetic tree, taking into account the size of the individual taxonomic categories of the oral microbiome among patients with $\mathrm{PD}$. The most numerous clusters were Bacteroidetes.

Oral microbiota in patients with PD included significantly more bacteria from genera Prevotella, Streptobacillus, Megaspheaera, and Lactobacillus. The healthy controls had oral microbiota more abundant in bacteria from genera Haemophilus, TM7, and Veillonella.

The most numerous species in the patients with PD were Prevotella histicola, Prevotella melaninogenica, and Porphyromonas gingivalis. The healthy controls had oral microbiota more abundant in bacteria from species Haemophilus parainfluenzae, Streptococcus sanguinis, Prevotella nanceiensis, and other species presented in Figure 6. 


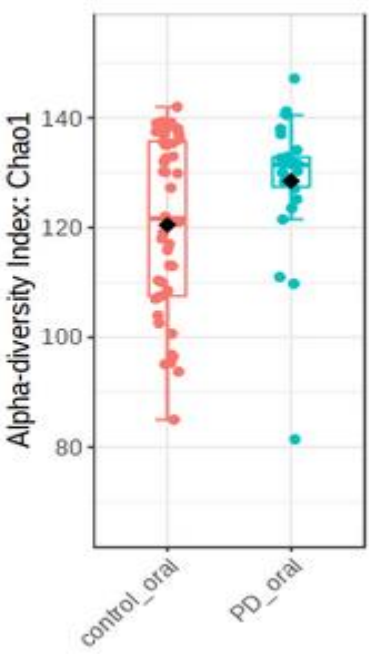

(a)

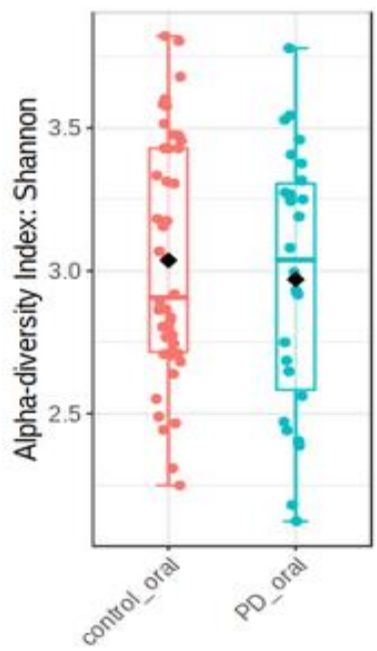

(c)

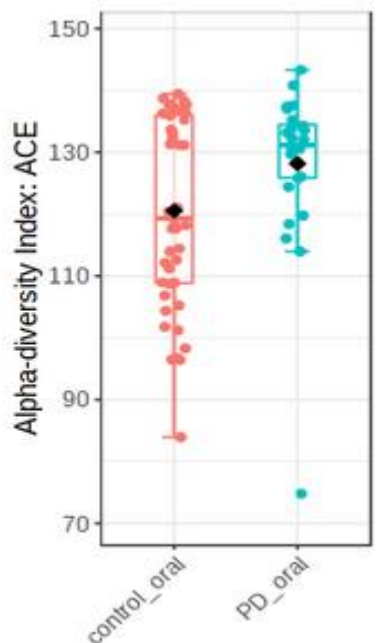

(b)
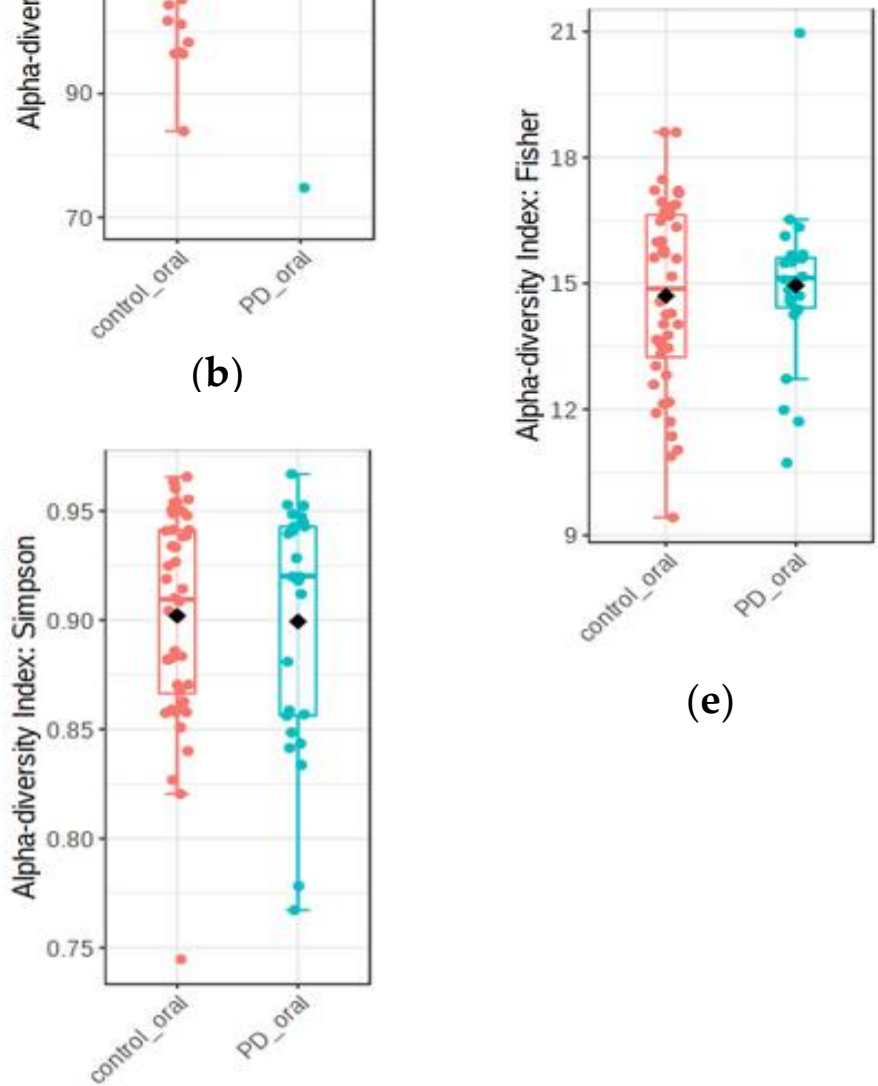

CLASS

由 control_oral

由 PD_oral

(e)

(d)

Figure 2. Comparisons of different alpha-diversity indices in patients with PD (PDG) and healthy controls (CG). (a) Chao1, $p$-value: $p<0.02$ between PDG and CS. (b) ACE $p<0.03$ between PDG and CG. (c) Shannon diversity index $(p<0.55)$ among patients with PD with different severity. (d) Simpson diversity index $(p<0.84)$ among patients with PD with different severity. (e) Fisher diversity index $(p<0.62)$ among patients with PD with different severity. 


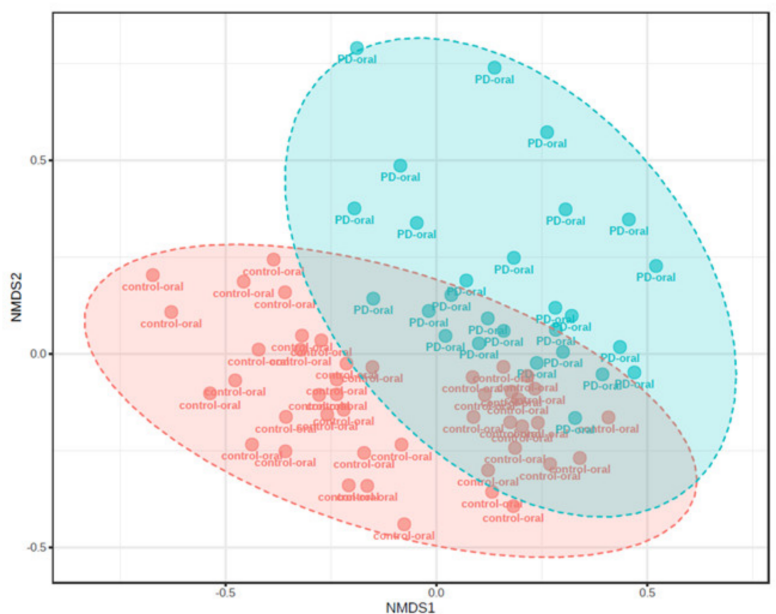

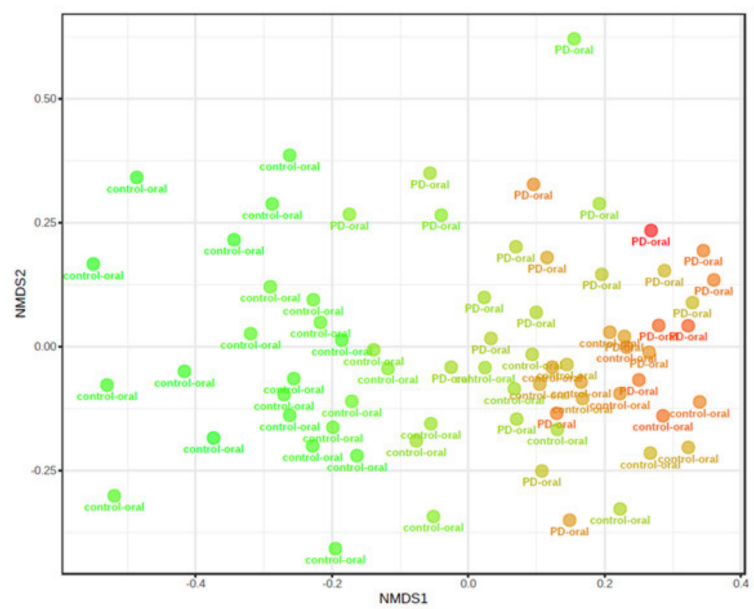

(b)

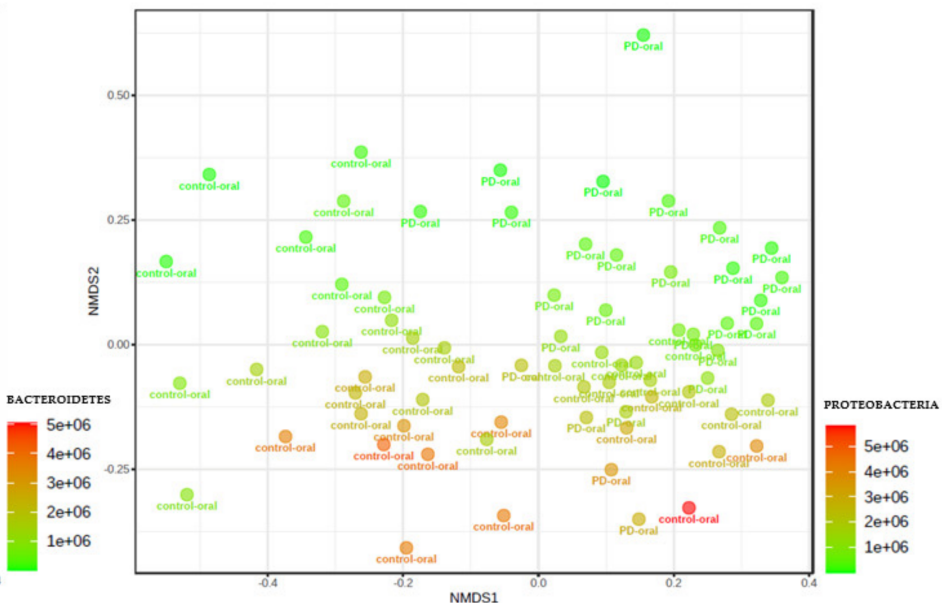

(c)

Figure 3. Visualization of oral microbial communities in patients with PD and healthy controls using non-metric multidimensional scheme. D stress values for all NMDS plots $=0.20593$. (a) Samples from patients with PD are indicated in blue and healthy controls are indicated in red. The $95 \%$ confidence intervals around the centroids for each group are shown. Oral microbiomes in patients with PD are significantly different from healthy controls, as demonstrated by analysis of similarity (ANOSIM); $\mathrm{R}=0.2372$ and $p<0.001$. (b) Identical NMDS plot, with samples color-coded by the abundance of bacteria from the phylum Bacteroidetes. (c) Identical NMDS plot, with samples color-coded by the abundance of bacteria from the phylum Proteobacteria. The strength and statistical significance were calculated based on a categorical variable found in the same mapping file. An R-value near 1 indicates dissimilarities between the groups, while 0 indicates no significant dissimilarities between the groups. 
(a)

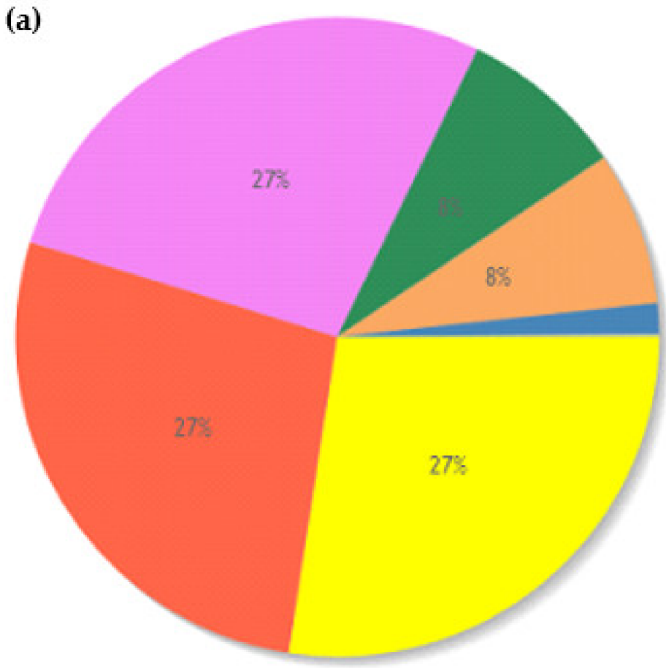

Actinobacteria

Firmicutes

Bacteroidetes

Fusobacteria

Proteobacteria

Patescibacteria
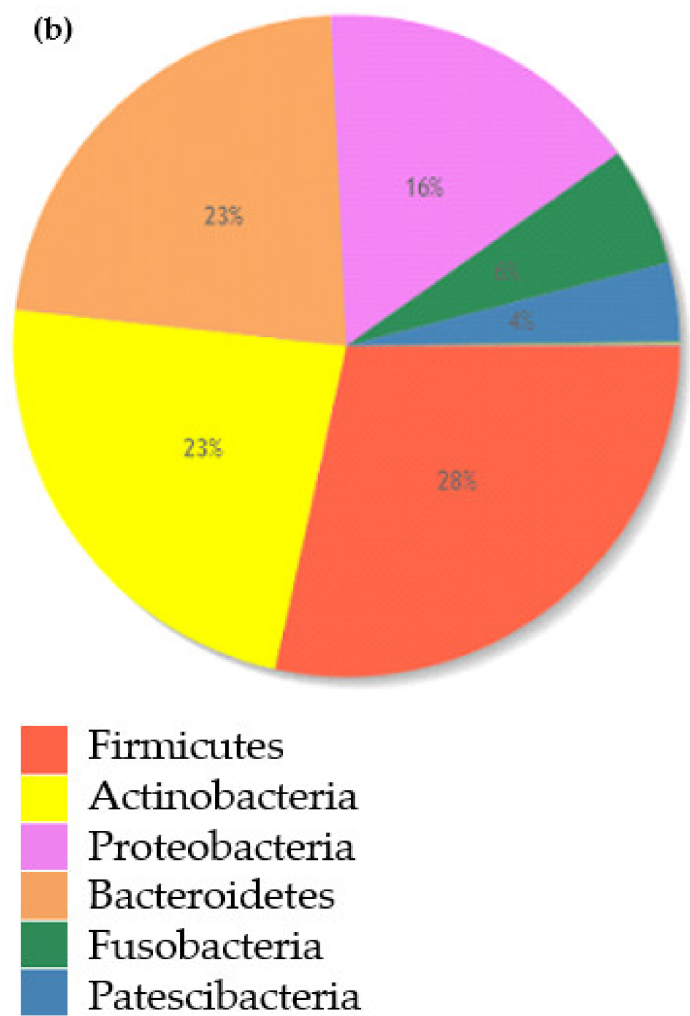

Figure 4. (a) Percentage distribution of the oral cavity microbiota of the patients with PD at the phylum level. (b) Percentage distribution of the microbiota of the oral cavity of the healthy controls at the phylum level.

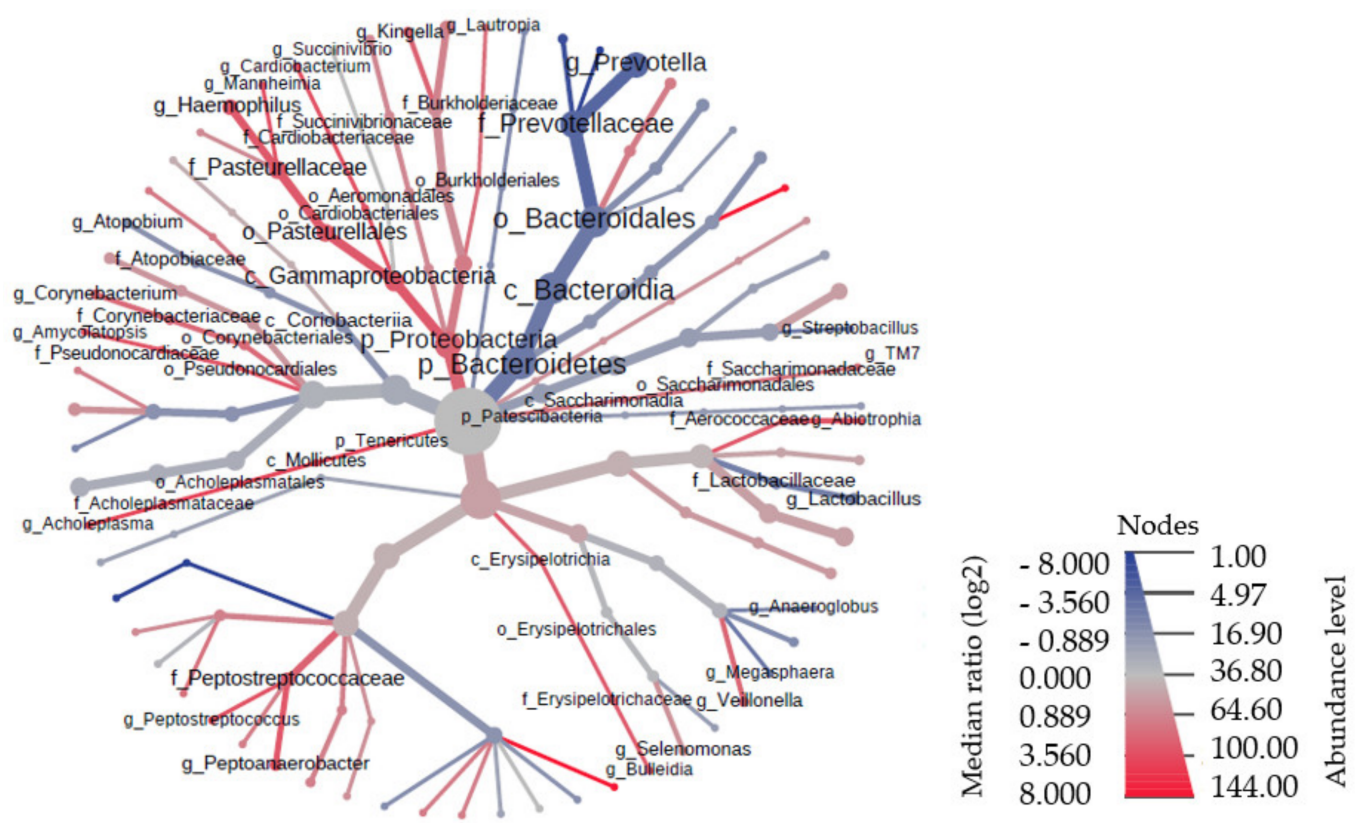

Figure 5. Phylogenetic tree of bacteria in the oral cavity of patients with PD. 


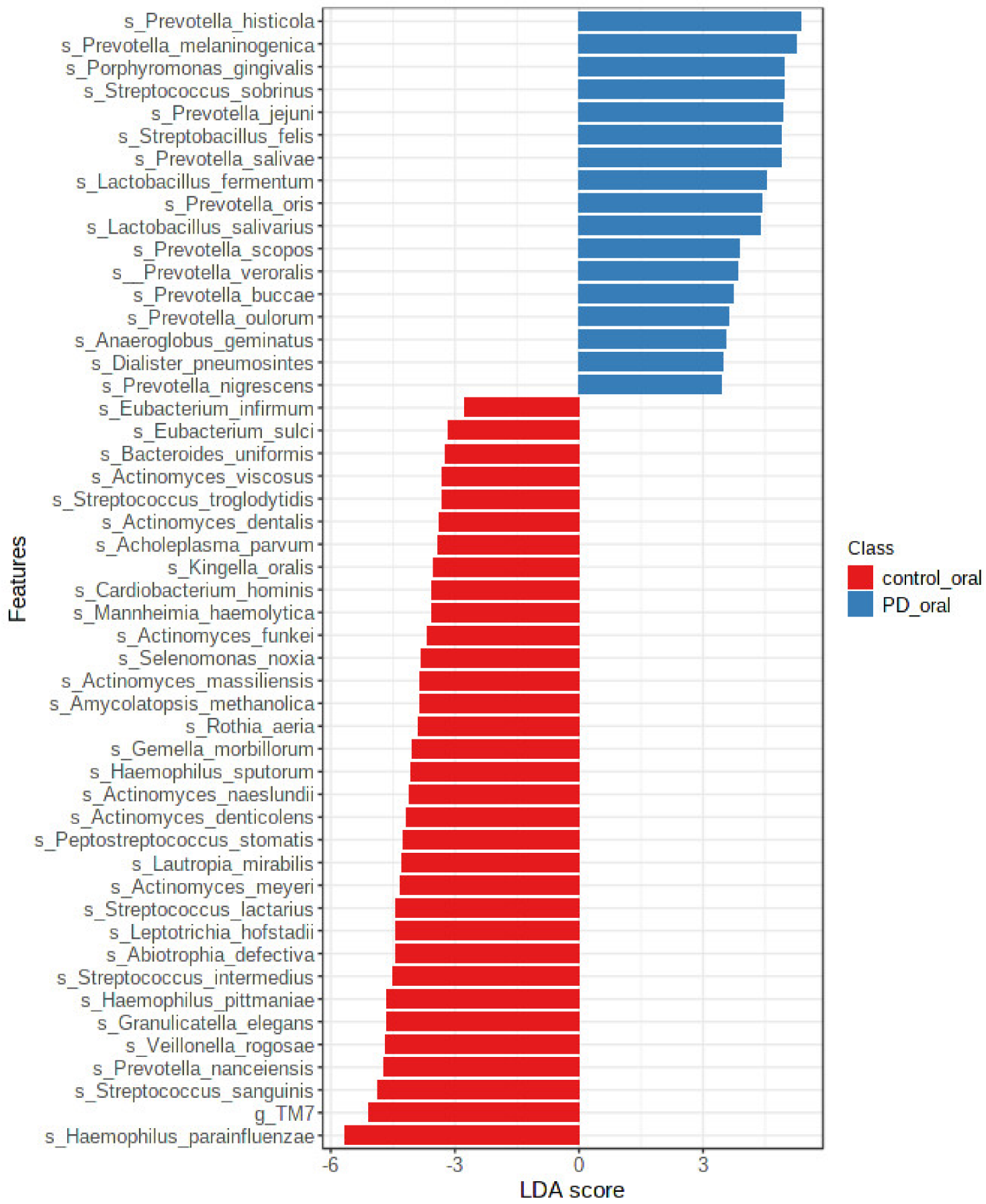

Figure 6. There are differences in oral microbiota between patients with PD and the healthy controls at the genre level.

\subsection{Correlation of Microbiota with Food Preferences}

Pearson correlation analysis revealed the species of bacteria which correlated with the frequency of some food products (Table 5). Several species of bacteria correlated positively with the frequency of consumption of several products. For example, the high abundance of Veillonella rogosae correlated with the high frequency of leaf vegetable consumption. The high abundance of Prevotella histicola correlated with a low frequency of red meat consumption. 
Table 5. The Pearson's correlation values show the relationship between bacteria and the frequency of consuming particular food products. Blue color means the most potent positive correlations, while red means the most potent negative correlations.

\begin{tabular}{|c|c|c|c|c|c|c|c|c|c|}
\hline & Cross Vegetables & Yellow Vegetables & Leaf Vegetables & Parsley & Root Vegetables & Tomatoes & Chicken & Soy & Peanuts \\
\hline Prevotella nanceiensis & -0.32 & 0.15 & 0.24 & -0.23 & 0.14 & 0.02 & -0.34 & -0.03 & -0.05 \\
\hline Haemophilus pittmaniae & -0.07 & 0.31 & -0.03 & -0.03 & 0.16 & 0.33 & -0.12 & -0.04 & -0.26 \\
\hline Streptococcus sanguinis & -0.18 & -0.20 & -0.16 & -0.10 & -0.14 & -0.24 & 0.23 & 0.03 & 0.15 \\
\hline Veillonella rogosae & 0.21 & 0.28 & 0.41 & 0.04 & 0.23 & 0.25 & -0.27 & -0.12 & -0.05 \\
\hline Porphyromonas gingivalis & -0.04 & -0.05 & 0.08 & -0.08 & 0.21 & 0.02 & 0.18 & -0.20 & -0.24 \\
\hline Streptobacillus felis & -0.34 & -0.04 & 0.06 & -0.10 & 0.13 & -0.10 & 0.04 & -0.24 & -0.13 \\
\hline Prevotella salivae & -0.10 & -0.22 & 0.03 & 0.19 & 0.13 & -0.07 & 0.11 & -0.03 & 0.05 \\
\hline Prevotella pallens & -0.12 & -0.07 & 0.10 & -0.02 & 0.17 & -0.19 & -0.14 & -0.03 & 0.19 \\
\hline Megasphaera micronuciformis & 0.10 & 0.20 & 0.22 & 0.21 & 0.30 & -0.01 & -0.05 & 0.08 & 0.06 \\
\hline Prevotella jejuni & -0.05 & -0.30 & 0.23 & 0.28 & 0.18 & -0.23 & 0.08 & 0.09 & 0.32 \\
\hline Prevotella histicola & 0.05 & -0.02 & 0.25 & 0.16 & 0.04 & 0.00 & -0.13 & 0.20 & 0.02 \\
\hline Prevotella melaninogenica & -0.29 & -0.52 & 0.00 & -0.09 & 0.01 & -0.13 & -0.09 & -0.16 & 0.20 \\
\hline \multirow[t]{2}{*}{ Streptococcus sobrinus } & 0.01 & 0.22 & 0.06 & -0.09 & -0.13 & -0.07 & -0.27 & -0.10 & -0.10 \\
\hline & Fruit & Apricots & Avocado and Olives & Dry Fruits & Fresh Fruits & Canned Fruits & Milk & Eggs & $\begin{array}{l}\text { Dark } \\
\text { Bread }\end{array}$ \\
\hline Prevotella nanceiensis & 0.07 & -0.23 & 0.03 & -0.04 & -0.08 & 0.07 & -0.20 & -0.40 & -0.13 \\
\hline Haemophilus pittmaniae & 0.19 & -0.31 & -0.04 & -0.07 & -0.01 & 0.12 & 0.13 & -0.11 & -0.20 \\
\hline Streptococcus sanguinis & -0.04 & -0.08 & -0.10 & 0.14 & -0.13 & -0.16 & 0.38 & -0.02 & 0.03 \\
\hline Veillonella rogosae & 0.19 & -0.20 & 0.02 & -0.08 & 0.14 & -0.06 & 0.17 & 0.14 & 0.13 \\
\hline Streptobacillus felis & 0.06 & 0.08 & -0.04 & -0.14 & 0.14 & -0.14 & -0.16 & -0.12 & -0.26 \\
\hline Prevotella salivae & 0.05 & 0.26 & 0.08 & 0.09 & 0.26 & 0.31 & 0.02 & -0.28 & -0.16 \\
\hline Prevotella pallens & -0.04 & 0.03 & 0.19 & 0.10 & -0.08 & 0.15 & -0.08 & -0.43 & -0.05 \\
\hline Megasphaera micronuciformis & 0.00 & 0.26 & 0.24 & 0.06 & 0.04 & 0.06 & -0.02 & -0.45 & -0.03 \\
\hline Prevotella jejuni & 0.09 & 0.09 & 0.21 & 0.29 & -0.03 & 0.13 & 0.19 & -0.22 & 0.10 \\
\hline Prevotella histicola & 0.05 & 0.38 & 0.01 & 0.13 & 0.38 & 0.18 & -0.18 & -0.25 & 0.04 \\
\hline Prevotella melaninogenica & 0.17 & 0.03 & -0.07 & 0.16 & 0.22 & 0.23 & 0.01 & -0.04 & -0.10 \\
\hline \multirow[t]{2}{*}{ Streptococcus sobrinus } & -0.26 & 0.03 & 0.11 & -0.21 & -0.36 & -0.42 & -0.10 & -0.07 & 0.26 \\
\hline & White Bread & Thick Groats & $\begin{array}{c}\text { Breakfast Cereal } \\
\text { Products }\end{array}$ & Butter & Margarine & Plant Oils & Red Meat & $\begin{array}{l}\text { White } \\
\text { Meat }\end{array}$ & Fish \\
\hline Prevotella nanceiensis & 0.10 & -0.20 & 0.25 & -0.17 & 0.15 & -0.22 & -0.28 & -0.18 & -0.15 \\
\hline Haemophilus pittmaniae & 0.18 & -0.19 & 0.34 & 0.04 & -0.02 & 0.02 & 0.05 & -0.31 & -0.14 \\
\hline Streptococcus sanguinis & 0.01 & -0.10 & -0.17 & 0.09 & -0.06 & 0.35 & -0.03 & 0.12 & 0.06 \\
\hline Veillonella rogosae & 0.04 & -0.16 & 0.19 & 0.12 & -0.13 & -0.31 & 0.07 & -0.11 & -0.12 \\
\hline
\end{tabular}


Table 5. Cont.

\begin{tabular}{|c|c|c|c|c|c|c|c|c|c|}
\hline Haemophilus parainfluenzae & -0.02 & 0.03 & 0.22 & -0.04 & -0.01 & -0.24 & -0.18 & -0.20 & -0.09 \\
\hline Porphyromonas gingivalis & 0.21 & -0.12 & 0.16 & 0.16 & -0.19 & 0.19 & 0.20 & -0.25 & -0.12 \\
\hline Streptobacillus felis & -0.26 & -0.20 & -0.11 & -0.35 & 0.31 & -0.23 & -0.33 & -0.03 & -0.16 \\
\hline Prevotella salivae & 0.00 & 0.07 & 0.02 & -0.04 & -0.02 & -0.18 & -0.26 & -0.19 & 0.00 \\
\hline Prevotella pallens & 0.13 & -0.16 & 0.00 & -0.09 & 0.06 & -0.22 & -0.15 & -0.02 & -0.03 \\
\hline Megasphaera micronuciformis & -0.07 & 0.16 & 0.01 & -0.17 & 0.10 & -0.11 & -0.34 & -0.25 & -0.05 \\
\hline Prevotella histicola & -0.17 & 0.33 & 0.02 & -0.32 & 0.27 & -0.08 & -0.44 & -0.13 & -0.17 \\
\hline Prevotella melaninogenica & 0.13 & -0.22 & 0.06 & 0.26 & -0.28 & 0.08 & 0.18 & -0.14 & -0.18 \\
\hline Streptococcus sobrinus & -0.08 & -0.10 & -0.25 & -0.28 & 0.31 & -0.07 & 0.07 & 0.10 & -0.06 \\
\hline & Juices & Sweetened Drinks & Coffee & Alcohol & Amount of Water & Frying & Cooking & Baking & Suffocation \\
\hline Prevotella nanceiensis & -0.19 & 0.03 & -0.47 & -0.26 & -0.06 & -0.36 & 0.03 & -0.20 & 0.04 \\
\hline Streptococcus sanguinis & -0.05 & 0.12 & 0.04 & -0.16 & -0.02 & 0.17 & -0.08 & 0.08 & -0.14 \\
\hline Veillonella rogosae & 0.07 & -0.01 & 0.06 & -0.04 & -0.10 & 0.04 & 0.00 & -0.17 & -0.31 \\
\hline Haemophilus parainfluenzae & -0.21 & -0.18 & & -0.21 & -0.03 & -0.37 & 0.03 & -0.51 & -0.65 \\
\hline Porphyromonas gingivalis & 0.16 & -0.02 & 0.09 & 0.01 & -0.24 & 0.17 & 0.02 & -0.09 & 0.11 \\
\hline Streptobacillus felis & 0.00 & -0.22 & -0.28 & -0.13 & 0.03 & -0.17 & & 0.15 & 0.06 \\
\hline Prevotella salivae & 0.03 & -0.09 & -0.29 & -0.24 & & -0.27 & -0.32 & 0.00 & 0.11 \\
\hline Prevotella pallens & -0.20 & -0.10 & -0.49 & -0.48 & -0.18 & -0.22 & -0.05 & -0.04 & 0.30 \\
\hline Megasphaera micronuciformis & -0.09 & -0.10 & -0.37 & -0.42 & -0.04 & -0.33 & -0.19 & 0.03 & 0.16 \\
\hline Prevotella jejuni & -0.17 & -0.17 & -0.29 & -0.36 & -0.30 & -0.12 & -0.32 & -0.04 & 0.01 \\
\hline Prevotella histicola & 0.02 & -0.21 & -0.23 & -0.25 & -0.03 & -0.31 & -0.14 & 0.02 & 0.02 \\
\hline Prevotella melaninogenica & -0.26 & -0.01 & -0.03 & -0.12 & -0.52 & 0.16 & -0.01 & -0.04 & -0.14 \\
\hline Streptococcus sobrinus & -0.17 & -0.07 & 0.10 & 0.16 & 0.36 & -0.11 & 0.00 & -0.01 & 0.09 \\
\hline
\end{tabular}




\section{Discussion}

Currently, microbiota (both intestinal and oral) is a popular subject in current research, and it seems to have a significant impact on multiple neurodegenerative diseases [27]. In the presented study, we attempted to correlate bacterial microbiota composition in the oral cavity of patients with PD and the influence of consumed components of the Western diet on the development of the disease. We decided to conduct this research to better understand a disorder which remains a significant challenge for modern medicine [28]. Additionally, our study was extended to compare patients with PD to healthy controls.

We used the 16S rRNA molecular technique to analyze oral microbiota, which allowed us to assess the diversity of microbiota and composition of microbiota present in a given environment by precisely sequencing the bacterial DNA. Additionally, we compared the dietary ingredients consumed by both groups to validate the presented outcomes. It is essential to notice that differences in microbiota composition can result from factors associated with diet. The oral cavity is the residency environment for more than 700 bacterial species. The microbiome is both hydrated and nourished by saliva. The $\mathrm{pH} 6.5$ to 7.5 and the $37^{\circ} \mathrm{C}$ temperature are conditions that enable pathogenic and mutualistic bacteria to coexist. In healthy individuals, the predominant oral microbial communities belong to Firmicutes (genus Streptococcus, family Veillonellaceae, genus Granulicatella), Proteobacteria (genera Neisseria, Haemophilus), Actinobacteria (genera Corynebacterium, Rothia, Actinomyces), Bacteroidetes (genera Prevotella, Capnocytophaga, and Porhyromonas), and Fusobacteria (genera Fusobacterium) [29]. The most numerous are Actinomyces, Capnocytophaga, Eikenella, Eubacteria, Fusobacterium, Haemophilus, Lactobacterium, Leptotrichia, Neisseria, Porphyromonas, Prevotella, Propionibacterium, Peptostreptococcus, Streptococcus, Staphylococcus, Veillonella, and Treponema [29,30]. Multiple factors such as tobacco and alcohol may interfere with the composition of the oropharynx microbiota and may lead to chronic inflammatory [31].

In this study, we showed statistically significant differences in the oral microbiome of patients with PD compared to healthy controls. The oral microbiome of patients with PD was characterized mainly by the high abundance of genera Prevotellaceae, Lactobacillaceae, Streptobacillaceae, and Lactobacillaceae, which are regular residents of the oral microflora. However, it has been reported that the genus Prevotella may also be pathogenic and prevalent in disease infections [32]. The increased abundance of Prevotella spp. Has been as a critical factor in developing persistent inflammation in the gut, causing mucosal dysfunction and systemic inflammation [32]. Systemic inflammation is related to the progression of different diseases, including PD.

Moreover, Streptococcus spp., which have also been increased in our study, were related previously to gastritis in patients without Helicobacter pylori infections [33]. This study also reported statistically different results related to food preferences between the patients with PD and the healthy controls. We noticed that, in patients with PD, the consumption of margarine, fish, red meat, avocado, olives, and cereal products was higher than in healthy controls. Deficient consumption was observed for thick groats, sweetened drinks, peanuts, dark bread, fruits, vegetables, and soy in the same group of patients with PD.

A study by $\mathrm{Qu}$ et al. found a consistent association between PUFA consumption and a lower risk of PD, while higher consumption of cholesterol and arachidonic acid was associated with an increased risk of $\mathrm{PD}$, and our results showed no difference between the consumption of these fats in the two groups [34]. Our research did not show statistical significance in protein consumption between the two groups. This is in contrast to the results from the study of Honglei et al., which confirmed the association of high dairy consumption with an increased risk of PD behavior, especially in men [35]. In this study, increased consumption of animal fats in patients with PD was observed [34]. Our research has shown a low consumption of nuts, vegetables, and fruits in patients with PD, which is associated with a decrease in resveratrol. According to Arbo et al.'s research, this substance has a neuroprotective effect, including regulating pro-apoptotic proteins that affect cell 
death or counteracting changes in the morphology of mitochondria and the potential of the mitochondrial membrane [36-39]. The relationship between bacteria of the oral cavity and food preferences revealed the highest, positive correlation of Veillonella rogosae with the high frequency of leaf vegetable consumption. The highest negative correlation was observed for Prevotella pallens and alcohol consumption. Partially, our results differ in the diversity of bacteria compared to other studies, for example, results reported by Pedro A.B Pereira et al., which distinguish more types of bacteria in patients such as Streptococcus, Haemophilus, Neisseria, and Veillonella [16]. In this study, the most numerous were Prevotella histicola, Prevotella melaninogenica, and Porphyromonas gingivalis. In another study by Rozas et al. the most numerous were Lactobacillus, Tannerella forsythia, and Prevotella intermedia [18]. The differences between the bacterial composition may depend on genetic factors, the inhabited area, type of diet, illnesses, stimulants, stress, or medications used by patients. This study revealed the perturbation in oral microbiota of patients with PD, which differed from those of the healthy controls. The microbial biomarkers that we discovered may contribute to the pathogenesis of PD. The results that we demonstrate in this report may be a critical window in PD and microbiota development, creating the opportunity for novel therapies in the future. Unlike genetics, the microbiota, oral microbiota seems to be an easy target of modifications throughout life. Understanding the interaction between the oral cavity microbiota composition and PD progression is very important in nutritional-based interventions, especially in high-risk groups.

The size of the study was determined based on advanced technologies for microbiota composition analysis, which are associated with the high cost of such research. This study is associated with several limitations. The most critical was that the study group was not particularly abundant, and a more extensive study cohort would yield more precise results. There is also a significantly high percentage (79.7\%) of people in the control group who have only primary or lower education, and this is essential to consider when evaluating their health literacy and priorities in choosing a diet. Moreover, the group of patients with PD included demented people, with a condition that could impact dental hygiene and oral microbiota composition.

Additionally, it is essential to note that the presented study is comparative, introducing potential bias. Therefore, it is essential to preserve caution when generalizing presented outcomes.

For researchers who would like to expand the research topic, we recommend including more diversified groups of patients, and they should present a broader spectrum of socioeconomic status and consider further education. Patients with PD should also be divided into more groups depending on the disease stage, which allows verification of whether the influence of the diet is similar for advanced PD patients. Moreover, we recommend analyzing the changes in the intestinal and nasal microbiome.

\section{Conclusions}

These findings suggest that oral microbiota could be associated with the development of PD. Firmicutes and Actinobacteria dominated the oral cavity microbiome in both examined groups. Patients with PD stand out with a higher concentration of phylum Bacteroidetes, and the healthy controls were recognizable because of the significant participation of Proteobacteria.

Author Contributions: Conceptualization: B.Z. and M.R.-B.; methodology: B.Z., T.M., M.W.-P., M.D. and Z.J.; software: B.Z. and M.P.; validation: B.Z., M.P. and M.R.-B.; formal analysis: M.R.B.; investigation: B.Z., T.M. and A.D.; resources: B.Z.; data curation: B.Z.; writing-original draft preparation: B.Z., T.S. and J.W.; writing—review and editing: M.R.-B.; visualization: B.Z.,M.P., T.S. and J.W.; supervision: M.R.-B.; project administration: B.Z.; funding acquisition: B.Z. and T.M. All authors have read and agreed to the published version of the manuscript. 
Funding: This research was funded by institutional funding of the Jagiellonian University Medical College and Ministry of Science and Higher Education; grant number MNiSW/2020/371/DIR/NN4, and grant number N41/DBS/000816.

Institutional Review Board Statement: The research was conducted in compliance with our department hospital's highest ethical standards, national guidelines, and the Helsinki Declaration. All protocols for the study were approved by the Ethics Committee of Jagiellonian University Medical College (approvement number 1072.6120.267.2019) and written informed consent was obtained from each of the subjects before enrollment.

Informed Consent Statement: Informed consent was obtained from all subjects involved in the study.

Conflicts of Interest: The authors declare no conflict of interest. The funders had no role in the study's design; in the collection, Analyses, or interpretation of data; in the writing of the manuscript; or in the decision to publish the results.

\section{References}

1. Lees, A.J.; Hardy, J.; Revesz, T. Parkinson's disease. Lancet 2009, 373, 2055-2066. [CrossRef]

2. Kalia, L.V.; Lang, A.E. Parkinson's disease. Lancet 2015, 386, 896-912. [CrossRef]

3. Obeso, J.A.; Stamelou, M.; Goetz, C.G.; Poewe, W.; Lang, A.E.; Weintraub, D. Past. Present. Future of Parkinson's disease: A special essay on the 200th Anniversary of the Shaking Palsy. Mov. Disord. 2017, 32, 1264-1310. [CrossRef]

4. Seidl, S.E.; Santiago, J.A.; Bilyk, H.; Potashkin, J.A. The emerging role ofnutrition in Parkinson's disease. Front. Aging Neurosci. 2014, 6, 36. [CrossRef]

5. Perez-Pardo, P.; Kliest, T.; Dodiya, H.B.; Broersen, L.M.; Garssen, J.; Keshavarzian, A.; Kraneveld, A.D. The gut-brain axis in Parkinson's disease:Possibilities for food-based therapies. Eur. J. Pharmacol. 2017, 817, 86-95. [CrossRef] [PubMed]

6. Rassmussen, H.; Piazza, B.R.; Forsyth, C.B.; Keshavarzian, A. Nutrition and gastrointestinal health as modulators of Parkinson's disease. In Pharma-Nutrition-AAPS Advances in the Pharmaceutical Sciences Series; Folkerts, G., Garssen, J., Eds.; Springer: New York, NY, USA, 2014; pp. 213-242. [CrossRef]

7. Mischley, L.K.; Lau, R.C.; Bennett, R.D. Role of diet and nutritionalsupplements in Parkinson's disease progression. Oxidative Med. Cell Longev. 2017, 2017, 6405278. [CrossRef]

8. Makki, K.; Deehan, E.C.; Walter, J.; Bäckhed, F. The impact of dietary fiber on gutmicrobiota in host health and disease. Cell Host Microbe. 2018, 23, 705-715. [CrossRef] [PubMed]

9. Christ, A.; Günther, P.; Lauterbach, M.A.R.; Duewell, P.; Biswas, D.; Pelka, K.; Scholz, C.-J.; Oosting, M.; Haendler, K.; Baßler, K.; et al. Western diet triggers NLRP3-dependent innate immune reprogramming. Cell 2018, 172, 162-175. [CrossRef] [PubMed]

10. Myles, I.A. Fast food fever: Reviewing the impacts of the Westerndiet onimmunity. Nutr. J. 2014, 13, 61. [CrossRef]

11. Maraki, M.I.; Yannakoulia, M.; Stamelou, M.; Stefanis, L.; Xiromerisiou, G.; Kosmidis, M.H.; Dardiotis, E.; Hadjigeorgiou, G.M.; Sakka, P.; Anastasiou, C.A.; et al. Mediterranean diet adherence is related to reduced probability of prodromal Parkinson's disease. Mov. Disord. 2019, 34, 48-57. [CrossRef]

12. Gao, X.; Cassidy, A.; Schwarzschild, M.A.; Rimm, E.B.; Ascherio, A. Habitual intake of dietary flavonoids and risk of Parkinson disease. Neurology 2012, 78, 1138-1145. [CrossRef]

13. Bousquet, M.; Calon, F.; Cicchetti, F. Impact of omega-3 fatty acids in Parkinson's disease. Ageing Res. Rev. 2011, 10, 453-463. [CrossRef] [PubMed]

14. Kamel, F.; Goldman, S.; Umbach, D.M.; Chen, H.; Richardson, G.; Barber, M.R.; Meng, C.; Marras, C.; Korell, M.; Kasten, M.; et al. Dietary fat intake, pesticide use, and Parkinson's disease. Park. Relat. Disord. 2013, 20, 82-87. [CrossRef]

15. Mihaila, D.; Donegan, J.; Barns, S.; LaRocca, D.; Du, Q.; Zheng, D.; Vidal, M.; Neville, C.; Uhlig, R.; Middleton, F.A. The oral microbiome of early stage Parkinson's disease and its relationship with functional measures of motor and non-motor function. PLoS ONE 2019, 14, e0218252. [CrossRef] [PubMed]

16. Pereira, P.A.B.; Aho, V.T.E.; Paulin, L.; Pekkonen, E.; Auvinen, P.; Scheperjans, F. Oral and nasal microbiota in Parkinson's disease. Parkinsonism Relat. Disord. 2017, 38, 61-67. [CrossRef]

17. Fleury, V.; Zekeridou, A.; Lazarevic, V.; Gaia, N.; Giannopoulou, C.; Genton, L. Oral Dysbiosis and Inflammation in Parkinson's Disease. J. Parkinsons Dis. 2021, 11, 619-631. [CrossRef]

18. Rozas, N.; Tribble, G.; Jeter, C. Oral Factors That Impact the Oral Microbiota in Parkinson's Disease. Microorganisms 2021, 9, 1616. [CrossRef] [PubMed]

19. Klindworth, A.; Pruesse, E.; Schweer, T.; Peplies, J.; Quast, C.; Horn, M.; Glöckner, F.O. Evaluation of general 16S ribosomal RNA gene PCR primers for classical and next-generation sequencing-based diversity studies. Nucleic Acids Res. 2013, 41, e1. [CrossRef] [PubMed]

20. Wang, Q.; Garrity, G.M.; Tiedje, J.M.; Cole, J.R. Naive Bayesian classifier for rapid assignment of rRNA sequences into the new bacterial taxonomy. Appl. Environ. Microbiol. 2007, 73, 5261-5267. [CrossRef]

21. Good, I.J. The population frequencies of species and the estimation of population parameters. Biometrika 1953, 40, 237-264. [CrossRef] 
22. Schloss, P.D.; Westcott, S.L.; Ryabin, T.; Hall, J.R.; Hartmann, M.; Hollister, E.B.; Lesniewski, R.A.; Oakley, B.B.; Parks, D.H.; Robinson, C.J.; et al. Introducing mothur: Open-Source, Platform-Independent, Community-Supported Software for Describing and Comparing Microbial Communities. Appl. Environ. Microbiol. 2009, 75, 7537-7541. [CrossRef]

23. Lozupone, C.; Knight, R. UniFrac: A New Phylogenetic Method for Comparing Microbial Communities. Appl. Environ. Microbiol. 2005, 71, 8228-8235. [CrossRef]

24. Segata, N.; Izard, J.; Waldron, L.; Gevers, D.; Miropolsky, L.; Garrett, W.S.; Huttenhower, C. Metagenomic biomarker discovery and explanation. Genome Biol. 2011, 12, R60. [CrossRef]

25. Chong, J.; Liu, P.; Zhou, G.; Xia, J. Using Microbiome Analyst for comprehensive statistical functional and meta-analysis of microbiome data. Nat. Protoc. 2020, 15, 799-821. [CrossRef]

26. Foster, Z.S.; Sharpton, T.J.; Grünwald, N.J. MetacodeR: An R package for visualization and manipulation of community taxonomic diversity data. PLoS Comput. Biol. 2016, 13, e1005404. [CrossRef]

27. Sarkar, S.R.; Banerjee, S. Gut microbiota in neurodegenerative disorders. J. Neuroimmunol. 2019, 328, 98-104. [CrossRef] [PubMed]

28. Jackson, A.; Forsyth, C.B.; Shaikh, M.; Voigt, R.M.; Engen, P.A.; Ramirez, V.; Keshavarzian, A. Diet in Parkinson's Disease: Critical Role for the Microbiome. Front. Neurol. 2019, 10, 1245. [CrossRef] [PubMed]

29. Zaura, E.; Keijser, B.J.F.; Huse, S.M.; Crielaard, W. Defining the healthy "core microbiome" of oral microbial communities BMC Microbiol. 2009, 9, 259. [CrossRef]

30. Lu, M.; Xuan, S.; Wang, Z. Oral microbiota: A new view of body health. Food Sci. Hum. Wellness 2019, 8, 8-15. [CrossRef]

31. Chattopadhyay, I.; Verma, M.; Panda, M. Role of Oral Microbiome Signatures in Diagnosis and Prognosis of Oral Cancer. Technol. Cancer Res. Treat. 2019, 18, 1533033819867354. [CrossRef]

32. Castellanos, J.F.-V.; Serrano-Villar, S.; Latorre, A.; Artacho, A.; Ferrús, M.L.; Madrid, N.; Moya, A. Altered metabolism of gut microbiota contributes to chronic immune activation in HIV-infected individuals. Mucosal Immunol. 2015, 8, 760-772. [CrossRef] [PubMed]

33. Nardone, G.; Compare, D. The human gastric microbiota: Is it time to rethink the pathogenesis of stomach diseases? United Eur. Gastroenterol. J. 2015, 3, 255-260. [CrossRef] [PubMed]

34. Sun, Q.; Qu, Y.; Chen, X.; Xu, M.-M. Relationship between high dietary fat intake and Parkinson's disease risk: A meta-analysis. Neural Regen. Res. 2019, 14, 2156-2163. [CrossRef]

35. Chen, H.; O’Reilly, E.; McCullough, M.L.; Rodriguez, C.; Schwarzschild, M.A.; Calle, E.E.; Thun, M.J.; Ascherio, A. Consumption of Dairy Products and Risk of Parkinson's Disease. Am. J. Epidemiol. 2007, 165, 998-1006. [CrossRef]

36. Arbo, B.D.; André-Miral, C.; Nasre-Nasser, R.G.; Schimith, L.E.; Santos, M.G.; Costa-Silva, D.; Muccillo-Baisch, A.L.; Hort, M.A. Resveratrol Derivatives as Potential Treatments for Alzheimer's and Parkinson's Disease. Front. Aging Neurosci. 2020, $12,103$. [CrossRef] [PubMed]

37. Zhang, H.; Bai, L.; He, J.; Zhong, L.; Duan, X.; Ouyang, L.; Zhu, Y.; Wang, T.; Zhang, Y.; Shi, J. Recent advances in discovery and development of natural products as source for anti-Parkinson's disease lead compounds. Eur. J. Med. Chem. 2017, 141, 257-272. [CrossRef] [PubMed]

38. Zhang, Y.; Li, Y.; Wang, Y.; Wang, G.; Mao, L.; Zhang, D.; Wang, J. Effects of resveratrol on learning and memory in rats with vascular dementia. Mol. Med. Rep. 2019, 20, 4587-4593. [CrossRef] [PubMed]

39. Feng, Y.; Liu, T.; Dong, S.-Y.; Guo, Y.-J.; Jankovic, J.; Xu, H.; Wu, Y.-C. Rotenone affects p53 transcriptional activity and apoptosis via targeting SIRT1 and H3K9 acetylation in SH-SY5Y cells. J. Neurochem. 2015, 134, 668-676. [CrossRef] [PubMed] 